\title{
ARTICLE
}

\section{Insufficient Self-Shielding Correction in VITAMIN-B6}

\author{
Chikara KONNO*, Kentaro OCHIAI, and Seiki OHNISHI \\ Japan Atomic Energy Agency, Tokai, Naka, Ibaraki 319-1195, Japan
}

\begin{abstract}
We carried out a simple benchmark calculation test with a multigroup cross-section library VITAMIN-B6 generated from ENDF/B-VI. The model of this test consisted of an iron sphere of $1 \mathrm{~m}$ in radius with an isotropic $20 \mathrm{MeV}$ neutron source in the center. Neutron spectra in the sphere were calculated with an Sn code ANISN and VITAMIN-B6 or FENDL/MG-1.1. A calculation with MCNP and ENDF/B-VI was carried out as a reference. The neutron spectra with ANISN and FENDL/MG-1.1 agreed with those with MCNP, while those with ANISN and VITAMIN-B6 were at most $50 \%$ different from those with MCNP. We uncovered that the discrepancy came from insufficient self-shielding correction due to the followings; 1) The smallest background cross section of ${ }^{56} \mathrm{Fe}$ in VITAMIN-B6 is 1.2) The weighting flux used in generating VITAMIN-B6 is not adequate. VITAMIN-B6 should be revised for adequate self-shielding correction.
\end{abstract}

\section{KEYWORDS: VITAMIN-B6, ANISN, self-shielding correction, background cross section, weighting flux}

\section{Introduction}

An AMPX master library VITAMIN-B6 ${ }^{1)}$ was released from ORNL in 1996. ENDF/B-VI release $3^{2)}$ data of 120 materials were processed to GENDF files (neutron 199 groups and gamma 42 groups) with the NJOY code ${ }^{3)}$. The GENDF files were converted to VITAMIN-B6 with the SMILER code $e^{4)}$. A multigroup library is produced with the SCAMPI $\operatorname{code}^{5)}$, where the self-shielding correction is carried out with the BONAMI code ${ }^{4)}$.

VITAMIN-B6 was verified through computational analyses of more than 30 benchmark experiments ${ }^{6)}$ and has been used widely in the world. However we encountered a strange result in a simple benchmark calculation test with VITAMIN-B6. Here we investigate reasons for the strange result in detail.

\section{Simple Benchmark Calculation Test}

The model of the simple benchmark calculation test consisted of a natural iron sphere of $1 \mathrm{~m}$ in radius with an isotropic neutron source of 17.732-19.96 MeV, which is the first group in VITMAIN-B6, in the center. Neutron spectra in the sphere were calculated with an Sn code ANISN $^{7)}$ and VITAMIN-B6. For comparison we also carried out an ANISN calculation with a multigroup library produced from FENDL/MG-1.1 ${ }^{8)}$, which is a MATXS file ${ }^{9)}$ for fusion reactors and iron data of which were taken from ENDF/B-VI, by using the TRANSX ${ }^{9}$ code. Moreover a calculation with MCNP4C ${ }^{10)}$ and ENDF/B-VI was carried out as a reference.

Figure 1 shows the calculated neutron spectra at the distance of $40 \mathrm{~cm}$ from the center of the iron sphere. The neutron spectrum with ANISN and FENDL/MG-1.1 agrees with that with MCNP, while the neutron spectrum below 100 $\mathrm{keV}$ with ANISN and VITAMIN-B6 is at most $50 \%$ larger than that with MCNP.

*Corresponding author, Tel. +81-29-282-6859, Fax. +81-29-282

-5709, E-mail: konno.chikara@jaea.go.jp
Figure 2 shows the calculated neutron spectra at the distance of $80 \mathrm{~cm}$ from the center of the iron sphere. The tendency is similar with that in Fig. 1, but the neutron spectrum with ANISN and VITAMIN-B6 is at most $30 \%$ larger below $100 \mathrm{keV}$ and by a half smaller from $100 \mathrm{keV}$ to $1 \mathrm{MeV}$ than that with MCNP.

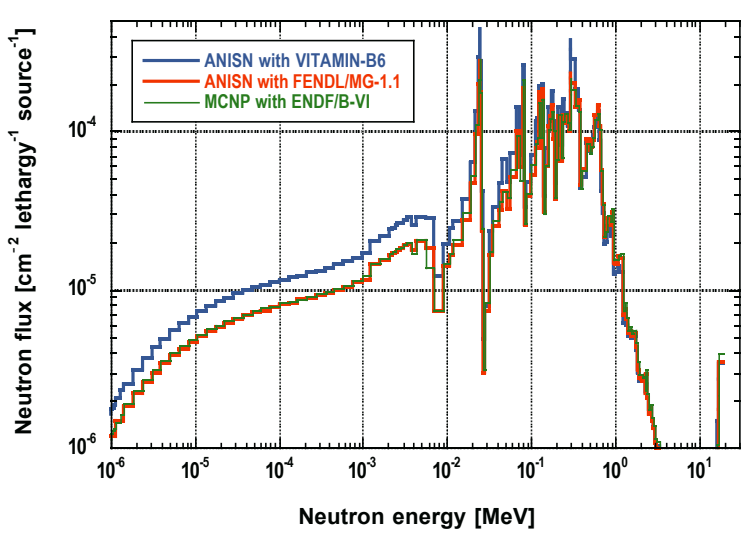

Fig. 1 Neuron spectra at $40 \mathrm{~cm}$ from center of iron sphere

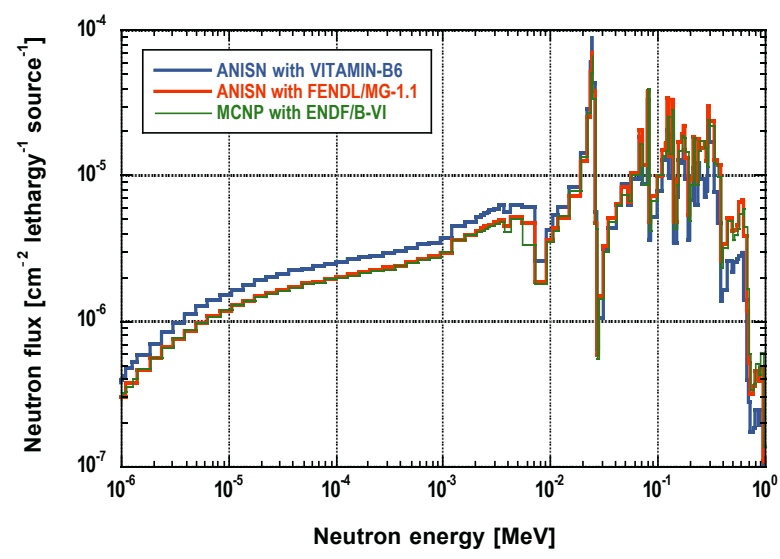

Fig. 2 Neuron spectra at $80 \mathrm{~cm}$ from center of iron sphere 


\section{Problems in VITAMIN-B6}

In order to investigate reasons for the above discrepancy in the neutron flux below $1 \mathrm{MeV}$, we compared VITAMIN-B6 with FENDL/MG-1.1 in detail.

\section{Background Cross Section Data}

The self-shielding correction in VITAMIN-B6 and FENDL/MG-1.1 is performed with the BONAMI code and the TRANSX code, respectively, by using the Bondarenko method $^{11)}$. In the Bondarenko method, the following weighting flux is used for averaging cross section data in a group,

$$
W_{\ell}(E)=\frac{C(E)}{\left[\sigma_{0}+\sigma_{t}(E)\right]^{\ell+1}},
$$

where $C(E)$ is a smooth part of the shape of the flux, $\sigma_{0}$ is called a background cross section, $\sigma_{t}(E)$ is a total cross section and $\ell$ is a Legendre order.

We found out that the prepared background cross section data for ${ }^{56} \mathrm{Fe}$ were different between VITAMIN-B6 and FENDL/MG-1.1; Bondarenko factors for self-shielding correction for the background cross sections of $10^{10}, 10^{5}, 10^{3}$, $10^{2}, 50,10$ and 1 barn are included in ${ }^{56} \mathrm{Fe}$ of VITAMIN-B6, while those of $10^{10}, 1000,100,10,3,1,0.3$ and 0.1 barn are included in ${ }^{56} \mathrm{Fe}$ of FENDL/MG-1.1. Natural iron requires Bondarenko factors for the background cross section of approximately 0.2 for ${ }^{56} \mathrm{Fe}$, but Bondarenko factors for the background cross section of 1 are used for self-shielding correction in VITAMIN-B6, which leads insufficient self-shielding correction, because the smallest background cross section is 1. On the contrary, the self-shielding correction for natural iron is adequate in FENDL/MG-1.1 because the smallest background cross section is 0.1 . The multigroup macroscopic total cross sections of natural iron are plotted in Fig. 3. The total cross section of VITAMIN-B6 is different from that of FENDL/MG-1.1. VITAMIN-B6 should include Bondarenko factors for the background cross section of 0.1 . Note that BUGLE96 ${ }^{12)}$ (a

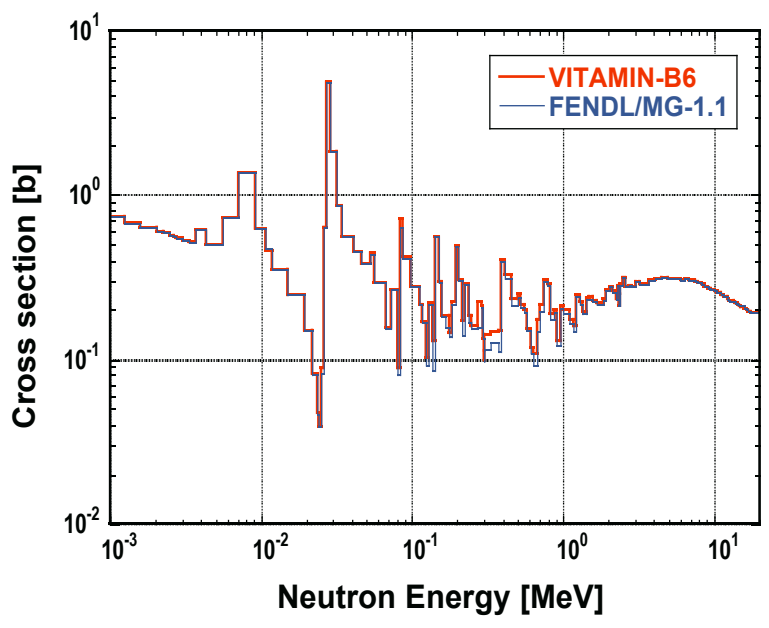

Fig. 3 Total cross section of natural iron broad group version of VITAMIN-B6) and HILO2 $\mathrm{K}^{13)}$ (data below $20 \mathrm{MeV}$ : VITAMIN-B6) also have the same problem. The same problem occurred in the FENDL/MG-1.0 $0^{14)}$ and it was revised in FENDL/MG-1.1 based on our comment ${ }^{15)}$. Other material data in VITAMIN-B6 should be also checked whether the prepared background cross sections are enough or not.

\section{Weighting Flux}

The weighting flux in Eq. 1 should be used for adequately averaging cross section data in a group. However the weighting fluxes for $\ell \geq 1$ used in VITAMIN-B6 and FENDL/MG-1.1 are the followings and are different from Eq. 1,

$$
\begin{aligned}
& W_{\ell \geq 1}(E)=W_{0}(E): \text { VITAMIN-B6, } \\
& W_{\ell \geq 1}(E)=W_{1}(E): \text { FENDL/MG-1.1. }
\end{aligned}
$$

Next influences of the difference between the weighting fluxes in Eqs. 2 and $\mathbf{3}$ were examined.

We have to remember that the $S_{N}$ multigroup cross sections for discrete ordinates codes are not the same as the $\mathrm{P}_{\mathrm{N}}$ multigroup cross sections which are included in VITAMIN-B6 and FENDL/MG-1.1. ${ }^{9}$ ) The relation between the $\mathrm{S}_{\mathrm{N}}$ and $\mathrm{P}_{\mathrm{N}}$ multigroup cross sections are following,

$$
\begin{aligned}
& \sigma_{\ell t g}^{P N}=\frac{\int_{g} \sigma_{t}(E) W_{\ell}(E) d E}{\int_{g} W_{\ell}(E) d E}, \\
& \sigma_{\ell g \leftarrow g^{\prime}}^{P N}=\frac{\int_{g^{\prime}} d E^{\prime} \int_{g} \sigma_{\ell}\left(E^{\prime} \rightarrow E\right) W_{\ell}\left(E^{\prime}\right) d E}{\int_{g^{\prime}} W_{\ell}\left(E^{\prime}\right) d E^{\prime}}, \\
& \sigma_{\ell g \leftarrow g^{\prime}}^{S N}=\sigma_{\ell g \leftarrow g^{\prime}}^{P N} \text { for } g \neq g^{\prime}, \\
& \sigma_{\ell g \leftarrow g}^{S N}=\sigma_{\ell g \leftarrow g}^{P N}-\left(\sigma_{\ell t g}^{P N}-\sigma_{0 t g}^{P N}\right)-\Delta_{g}^{N}, \\
& \sigma_{g}^{S N}=\sigma_{0 t g}^{P N}-\Delta_{g}^{N},
\end{aligned}
$$

where $\sigma_{\ell}\left(E^{\prime} \rightarrow E\right)$ is a scattering cross section and $\Delta_{g}^{N}$ can be chosen to minimize the effects of truncating the Legendre expansion at $\ell=N$. " $\Delta_{g}^{N}=0$ " is usually chosen, which is called "Consistent-P approximation". In this case, Eqs. 7 and 8 become

$$
\begin{aligned}
& \sigma_{\ell g \leftarrow g}^{S N}=\sigma_{\ell g \leftarrow g}^{P N}-\left(\sigma_{\ell t g}^{P N}-\sigma_{0 t g}^{P N}\right), \\
& \sigma_{g}^{S N}=\sigma_{0 t g}^{P N} .
\end{aligned}
$$

The difference between the $\mathrm{S}_{\mathrm{N}}$ and $\mathrm{P}_{\mathrm{N}}$ multigroup cross sections is only in Eq. 9. The term in parentheses of the right hand side in Eq. 9 is zero in VITAMIN-B6 because of Eq. 2, which leads $\sigma_{\ell g \leftarrow g}^{S N}=\sigma_{\ell g \leftarrow g}^{P N}$. However it is not always zero in FENDL/MG-1.1 because of Eq. 3, which leads $\sigma_{\ell g \leftarrow g}^{S N} \neq \sigma_{\ell g \leftarrow g}^{P N}$. Figure 4 shows the $\mathrm{P}_{1}$ coefficient of ingroup scattering cross section for natural iron. The $\mathrm{P}_{1}$ coefficient of the ingroup $\mathrm{S}_{\mathrm{N}}$ scattering cross section $\sigma_{\ell g \leftarrow g}^{S N}$ in VITAMIN-B6 is different from that in FENDL/MG-1.1 in the resonance energy region of natural iron. Note that the 


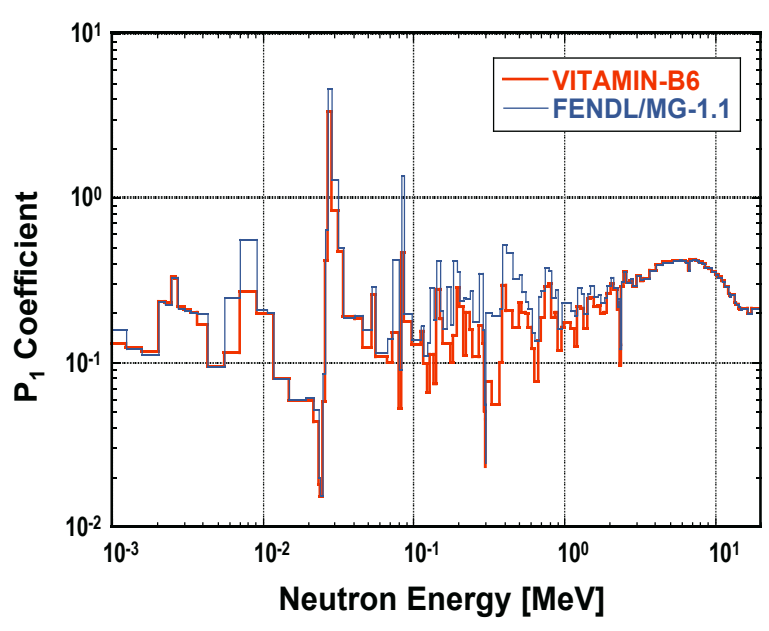

Fig. $4 \mathrm{P}_{1}$ coefficient of ingroup $\mathrm{S}_{\mathrm{N}}$ scattering cross section

ingroup $\mathrm{S}_{\mathrm{N}}$ scattering cross section $\sigma_{\ell g \leftarrow g}^{S N}$ in VITAMIN-B6 is almost the same as that in FENDL/MG-1.1 in the no-resonance region, because the weighting flux has a smooth shape in the no-resonance region.

The weighting flux for $\ell \geq 2$ in Eq. 3 is different from that in Eq. 1, but its influence is considered to be not so large because the neutron spectra calculated with ANISN and FENDL/MG-1.1 are similar to those with MCNP as shown in Figs. 1 and 2.

\section{Influences of Background Cross Section Data and Weighting Flux in VITAMIN-B6}

In order to examine influences due to the background cross section data and the weighting flux in VITAMIN-B6, three test MATXS files with the following conditions were produced from ENDF/B-VI by using NJOY99;

A) $\sigma_{0} \geq 1, W_{\ell}=W_{0}$ (the same condition as that in

VITAMIN-B6)

B) $\sigma_{0} \geq 1, W_{\ell}=W_{1}$ (revised weighting flux)

C) $\sigma_{0} \geq 0.1, W_{\ell}=W_{1}$ (the same condition as that in

FENDL/MG-1.1)

ANISN calculations with multigroup libraries produced from these MATXS files by using TRANSX were carried out. Figures 5 and 6 show the results. The neutron spectra with the case A) MATXS file are almost the same as those with VITAMIN-B6. The neutron spectra with the case B) MATXS file are improved, but not completely. On the contrary, the neutron spectra with the case C) MATXS file are almost the same as those with FENDL/MG-1.1. It is demonstrated that the difference between the neutron spectra with VITAMIN-B6 and FENDL/MG-1.1 comes from both the inadequate background cross section data and weighting flux in VITAMIN-B6.

\section{Processing Flow}

Figure 7 shows the flow of processing nuclear data for VITAMIN-B6 and FENDL/MG-1.1. At the first step the NJOY code is used for both VITAMIN-B6 and

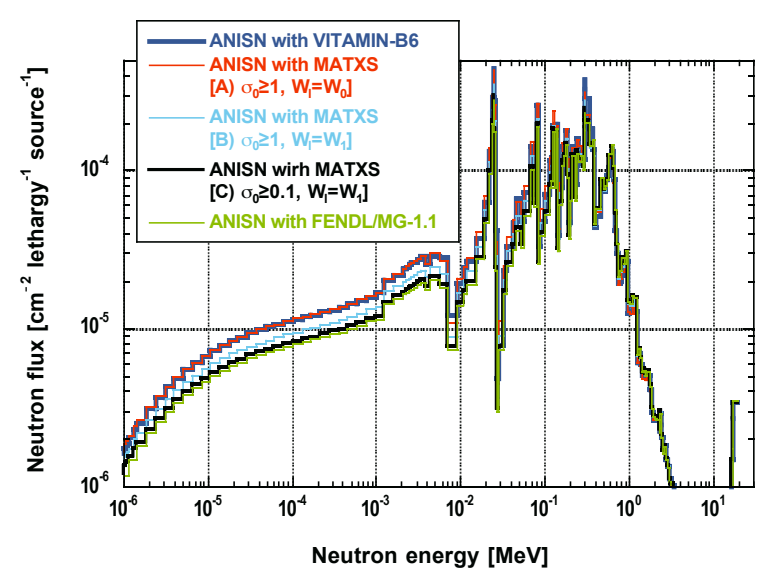

Fig. 5 Neuron spectra at $40 \mathrm{~cm}$ from center of iron sphere

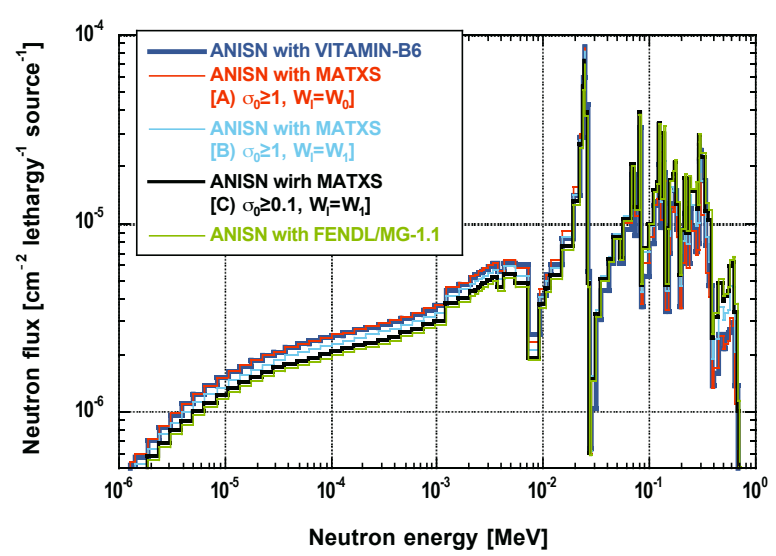

Fig. 6 Neuron spectra at $80 \mathrm{~cm}$ from center of iron sphere

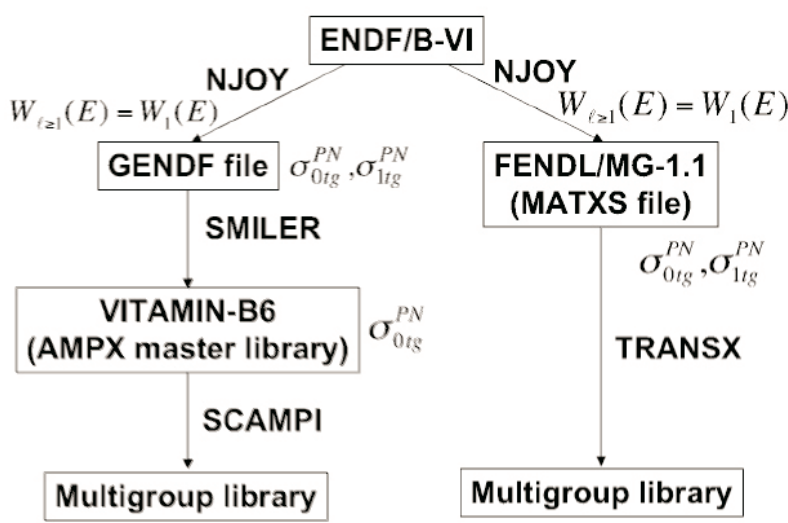

Fig. 7 Processing flow in VITAMIN-B6 and FENDL/MG-1.1

FENDL/MG-1.1. However the SMILER code omits the $\sigma_{1 t g}^{P N}$ in VITAMIN-B6 at the second step because the AMPX master format cannot include $\sigma_{1 t g}^{P N}$. Thus other multigroup libraries in the AMPX master format, e.g. BUGLE96 and HILO2K, also have the same problem for the weighting flux as VITAMIN-B6.

\section{Conclusion}

We carried out a simple benchmark calculation test with VITAMIN-B6 generated from ENDF/B-VI. The model of 
this test consisted of an iron sphere of $1 \mathrm{~m}$ in radius with an isotropic $20 \mathrm{MeV}$ neutron source in the center. The neutron spectra calculated with ANISN and FENDL/MG-1.1 agreed with those with MCNP, while those with ANISN and VITAMIN-B6 were at most $50 \%$ different from those with MCNP.

We examined VITAMIN-B6 in detail in order to investigate reasons for the discrepancy. It was found out that VITAMIN-B6 had two problems :

1) The smallest background cross section of ${ }^{56} \mathrm{Fe}$ in VITAMIN-B6 is 1, which is larger than that required for natural iron.

2) The weighting flux used in generating VITAMIN-B6 is not adequate.

These problems caused insufficient self-shielding correction, which leaded the discrepancy between neutron spectra with VITAMIN-B6 and FENDL/MG-1.1. VITAMIN-B6 should be revised based on this study. BUGLE96 and HILO2K also have the same problems.

\section{References}

1) J.E. White, R.Q. Wright, D.T. Ingersoll, R.W. Roussin, N.M. Greene, R.E. MacFarlane, "VITAMIN-B6: a fine-group cross section library based on ENDF/B-VI for radiation transport applications," Proc. of Int. Conf. on Nuclear Data for Science and Technology, Gatlinburg, Tennessee, May 9-13, 1994, p. 733 (1994).

2) P.F. Rose, "ENDF/B-VI summary documentation," BNL-NCS-17541 (ENDF-201) (1991).

3) R.E MacFarlane and D.W. Muir, "The NJOY nuclear data processing system, version 91," LA-12740-M (1994).

4) "SCALE: A Modular Code System for Performing Standardized Computer Analyses for Licensing Evaluation," NUREG/CR-0200, Rev.4 (ORNL/NUREG/CSD-2/R4), Vols. I-III (1995).
5) S. M. Bowman, "OFFSCALE: A PC Input Processor for the SCALE Code System Volume 1: The CSASIN Processor for the SCAMPI Code System," NUREG/CR-6182, Vol. 1 (ORNL/TM-12663/V1) (1995).

6) D.T. Ingersoll, J.E. White, R.Q. Wright, H.T. Hunter, C.O. Slater, N.M. Greene, R.E. MacFarlane, R.W. Roussin, "Production and Testing of the VITAMIN-B6 Fine-Group and the BUGLE-93 Broad-Group Neutron/Photon Cross-Section Libraries Derived from ENDF/B-VI Nuclear Data," ORNL-6795, NUREG/CR-6214 (1995).

7) "DOORS3.2a: One, two- and three-dimensional discrete ordinates neutron/photon transport code system," RSICC CODE PACKAGE CCC-650 (2007).

8) H. Wienke, S. Ganesan, IAEA-NDS-169, Rev. 3 (1996).

9) R.E. MacFarlane, "TRANSX 2: a code for interfacing MATXS cross-section libraries to nuclear transport codes, LA-12312-MS (1993).

10) J.F. Briesmeister (Ed.), "MCNP - a general Monte Carlo n-particle transport code, version 4C," LA-13709-M (2000).

11) I.I. Bondarenko (Ed.), "Group constants for nuclear reactor calculations," Consultants Bureau, New York, (1964).

12) J.E. White, D.T. Ingersoll, C.O. Slater, R.W. Roussin, "BUGLE-96: A Revised Multigroup Cross Section Library for LWR Applications Based on ENDF/B-VI Release 3," Proc. of Radiation Protection \& Shielding Topical Meeting, April 21-25, 1996, Falmouth, MA, (1996).

13) R.A. Lillie, F.X. Gallmeier, "HILO2k : A Coupled Neutron-Photon Transport Cross-Section Library for Neutron Energies up to $2000 \mathrm{MeV}$," Proc. 4th Int. Top. Meet. Nucl. Applicat. Accel. Tech., Washington, DC, p.302 (2000).

14) S. Ganesan, H. Wienke, "FENDL/MG-1.0," IAEA(NDS)-129, Rev.2 (1995).

15) C. Konno, F. Maekawa, M. Wada, "1.6 Influence of insufficient backgroud cross section of some nuclei in FEND/MG-1.0," JAERI-Review 96-012, p. 22 (1996). 